\title{
Charmed hadron production via equal-velocity quark combination in ultrarelativistic heavy ion collisions
}

\author{
Rui-Qin Wang, ${ }^{1, *}$ Jun Song, ${ }^{2, \dagger}$ Feng-Lan Shao $\odot,{ }^{1,+}$ and Zuo-Tang Liang ${ }^{3, \S}$ \\ ${ }^{1}$ School of Physics and Physical Engineering, Qufu Normal University, Shandong 273165, China \\ ${ }^{2}$ Department of Physics, Jining University, Shandong 273155, China \\ ${ }^{3}$ Key Laboratory of Particle Physics and Particle Irradiation (MOE), Institute of Frontier and Interdisciplinary Science, \\ Shandong University, Qingdao, Shandong 266237, China
}

(Received 5 November 2019; accepted 13 April 2020; published 12 May 2020)

\begin{abstract}
Recent data on the production of $D$ mesons and $\Lambda_{c}^{+}$baryons in heavy ion collisions at the BNL Relativistic Heavy Ion Collider and the CERN Large Hadron Collider exhibit a number of striking characteristics such as enhanced yield ratios $D_{s}^{+} / D^{0}$ and $\Lambda_{c}^{+} / D^{0}$ and their transverse momentum dependencies. In this article, we derive the momentum dependence of open charm mesons and singly charmed baryons produced in ultrarelativistic heavy ion collisions via the equal-velocity quark combination. We present analytic expressions and numerical results of yield ratios and compare them with the experimental data available. We make predictions for other charmed hadrons.
\end{abstract}

DOI: 10.1103/PhysRevC.101.054903

\section{INTRODUCTION}

In ultrarelativistic heavy ion collisions, heavy quarks and antiquarks are produced predominantly via initial hard scatterings and then experience the entire evolution of a violently interacting medium of deconfined quarks and gluons-the quark-gluon plasma (QGP). These heavy flavor quarks and antiquarks interact strongly with constituents of the QGP medium [1,2], exchange energy and momentum in the partonic evolution process [3-5], and combine with them to form heavy flavor hadrons that can be observed experimentally. Therefore, the production of heavy flavor hadrons is usually believed to play a very special role in studying the hadronization mechanism and properties of the QGP matter [6-11].

Recently, experimental data on open charm mesons and $\Lambda_{c}^{+}$baryons with improved precision and extended transverse momentum $\left(p_{T}\right)$ coverage at the BNL Relativistic Heavy Ion Collider (RHIC) and the CERN Large Hadron Collider (LHC) have been published [12-18]. These data show indeed a number of fascinating features. The most striking ones might be the enhancement of the strange to nonstrange meson yield ratio $D_{s}^{+} / D$ and that of the baryon-to-meson ratio $\Lambda_{c}^{+} / D^{0}$. It

\footnotetext{
*wangruiqin2010@mail.sdu.edu.cn

†songjun2011@jnxy.edu.cn

¥shaofl@mail.sdu.edu.cn

§liang@sdu.edu.cn
}

Published by the American Physical Society under the terms of the Creative Commons Attribution 4.0 International license. Further distribution of this work must maintain attribution to the author(s) and the published article's title, journal citation, and DOI. Funded by $\mathrm{SCOAP}^{3}$. has been observed $[15,16]$ that the $D_{s}^{+} / D$ ratio in ultrarelativistic heavy ion collisions is much higher than predictions of fragmentation models $[19,20]$. The $\Lambda_{c}^{+} / D^{0}$ ratio exhibits also a very strong enhancement trend at the intermediate- $p_{T}$ area not only in RHIC Au-Au and LHC Pb-Pb collisions but also in $p p$ and $p-\mathrm{Pb}$ reactions at the extremely high energies available at LHC [18]. They are much higher than the predictions of perturbative QCD and event generators based on fragmentation models [18,21].

Theoretically, much effort has also been put into the hadronization mechanism of charm quarks in high-energy heavy ion collisions [1,22-33]. It seems in particular that the coalescence or (re)combination mechanism should play an irreplaceable role in describing the production of charmed hadrons with low and intermediate transverse momenta [1,2432]. In this connection, we note in particular that the quark combination model under equal-velocity combination (hereafter referred to as EVC) [34] provides a very simple and elegant way to describe the $p_{T}$ dependence of production rates of hadrons and has been successfully applied [35,36] to $p p$ and $p-\mathrm{Pb}$ collisions to describe the enhancement of $\Lambda_{c}^{+} / D^{0}$. It is therefore interesting to see whether it can also be applied to heavy ion collisions to describe the heavy flavor hadron production as well.

In this article, we apply the quark combination via EVC to ultrarelativistic heavy ion collisions to study the production of open charm $D$ mesons and singly charmed baryons in the low- and intermediate- $p_{T}$ regions. We present the detailed derivations and give analytic expressions for $p_{T}$ dependencies of ratios of production rates of different hadrons. We compare the results with the experimental data available $[12,14,15]$ and make predictions for other types of hadrons.

The rest of the article is organized as follows. In Sec. II, we present the derivation of the momentum-dependent 
production of singly charmed hadrons in the quark combination model via EVC in ultrarelativistic heavy ion collisions. We present in particular the analytic expressions for two kinds of yield ratios measured by RHIC and LHC experiments [14-17] as the function of $p_{T}$ and discuss their qualitative properties. In Sec. III, we apply the results obtained in Sec. II to Au-Au collisions at $\sqrt{s_{\mathrm{NN}}}=200 \mathrm{GeV}$. In Sec. IV, we present a summary.

\section{THE CHARMED HADRON PRODUCTION IN THE QUARK COMBINATION VIA EVC}

The basic idea of the quark combination mechanism and formulas for the momentum dependence have been presented in many different literatures. An example of the derivation for light flavor hadrons starting from the general formulas has been given in Ref. [37] where $p_{T}$-integrated yield correlations have been calculated. Here, in this section, for explicitness, we just follow the same procedure as that in Ref. [37] and present the results of momentum dependence for singly charmed hadrons. After that, we present the corresponding results obtained under EVC.

\section{A. The general formalism}

To derive the momentum-dependent production rates of mesons and baryons in ultrarelativistic heavy ion collisions, just as in Ref. [37] we start with a color-neutral quarkantiquark system with $N_{q_{i}}$ quarks of flavor $q_{i}$ and $N_{\bar{q}_{i}}$ antiquarks of flavor $\bar{q}_{i}$ and suppose they hadronize via the quark combination mechanism. The momentum distributions $f_{M_{j}}\left(p ; N_{q_{i}}, N_{\bar{q}_{i}}\right)$ and $f_{B_{j}}\left(p ; N_{q_{i}}, N_{\bar{q}_{i}}\right)$ for the directly produced meson $M_{j}$ and baryon $B_{j}$ are given by

$$
\begin{aligned}
f_{M_{j}}\left(p ; N_{q_{i}}, N_{\bar{q}_{i}}\right)= & \sum_{\bar{q}_{1} q_{2}} \int d p_{1} d p_{2} N_{\bar{q}_{1} q_{2}} f_{\bar{q}_{1} q_{2}}^{(n)}\left(p_{1}, p_{2} ; N_{q_{i}}, N_{\bar{q}_{i}}\right) \\
& \times \mathcal{R}_{M_{j}, \bar{q}_{1} q_{2}}\left(p, p_{1}, p_{2} ; N_{q_{i}}, N_{\bar{q}_{i}}\right), \\
f_{B_{j}}\left(p ; N_{q_{i}}, N_{\bar{q}_{i}}\right)= & \sum_{q_{1} q_{2} q_{3}} \int d p_{1} d p_{2} d p_{3} \\
& \times N_{q_{1} q_{2} q_{3}} f_{q_{1} q_{2} q_{3}}^{(n)}\left(p_{1}, p_{2}, p_{3} ; N_{q_{i}}, N_{\bar{q}_{i}}\right) \\
& \times \mathcal{R}_{B_{j}, q_{1} q_{2} q_{3}}\left(p, p_{1}, p_{2}, p_{3} ; N_{q_{i}}, N_{\bar{q}_{i}}\right),
\end{aligned}
$$

where $f_{\bar{q}_{1} q_{2}}^{(n)}$ and $f_{q_{1} q_{2} q_{3}}^{(n)}$ are normalized joint momentum distributions for $\bar{q}_{1} q_{2}$ and $q_{1} q_{2} q_{3}$, respectively; $N_{\bar{q}_{1} q_{2}}=N_{\bar{q}_{1}} N_{q_{2}}$ is the number of $\bar{q}_{1} q_{2}$ pairs; $N_{q_{1} q_{2} q_{3}}$ is the number of $q_{1} q_{2} q_{3}$ clusters in the system, and it takes $N_{q_{1}} N_{q_{2}} N_{q_{3}}$ for $q_{1} \neq q_{2} \neq q_{3}$, $N_{q_{1}}\left(N_{q_{1}}-1\right) N_{q_{3}}$ for $q_{1}=q_{2} \neq q_{3}$, and $N_{q_{1}}\left(N_{q_{1}}-1\right)\left(N_{q_{1}}-2\right)$ for $q_{1}=q_{2}=q_{3}$; and kernel functions $\mathcal{R}_{M_{j}, \bar{q}_{1} q_{2}}$ and $\mathcal{R}_{B_{j}, q_{1} q_{2} q_{3}}$ stand for the probability density for a $\bar{q}_{1} q_{2}$ pair with momenta $p_{1}$ and $p_{2}$ to combine into a meson $M_{j}$ of momentum $p$ and that for a $q_{1} q_{2} q_{3}$ cluster with $p_{1}, p_{2}$, and $p_{3}$ to combine into a baryon $B_{j}$ of $p$, respectively.

Just as discussed in Ref. [37], $\mathcal{R}_{M_{j}, \bar{q}_{1} q_{2}}$ and $\mathcal{R}_{B_{j}, q_{1} q_{2} q_{3}}$ carry the kinematical and dynamical information of the quark combination. Their precise forms cannot be derived from the first principles due to their complicated nonperturbative nature. Nevertheless, they are constrained by a number of symmetry laws and rules such as the momentum conservation, constraints due to intrinsic quantum numbers such as spin and flavor, the requirement of the hadronization unitarity so that the production of all the open and hidden charm hadrons should exhaust all charm quarks and antiquarks in the system, and the meson-baryon production competition, and so on. To take these constraints into account explicitly, we rewrite them in the following forms:

$$
\begin{aligned}
& \mathcal{R}_{M_{j}, \bar{q}_{1} q_{2}}\left(p, p_{1}, p_{2} ; N_{q_{i}}, N_{\bar{q}_{i}}\right) \\
& =C_{M_{j}} \mathcal{R}_{\bar{q}_{1} q_{2}}^{(f)} \mathcal{R}_{M, \bar{q}_{1} q_{2}}\left(p_{1}, p_{2} ; N_{q_{i}}, N_{\bar{q}_{i}}\right) \delta\left(p_{1}+p_{2}-p\right), \\
& \mathcal{R}_{B_{j}, q_{1} q_{2} q_{3}}\left(p, p_{1}, p_{2}, p_{3} ; N_{q_{i}}, N_{\bar{q}_{i}}\right) \\
& =C_{B_{j}} \mathcal{R}_{q_{1} q_{2} q_{3}}^{(f)} \mathcal{R}_{B, q_{1} q_{2} q_{3}}\left(p_{1}, p_{2}, p_{3} ; N_{q_{i}}, N_{\bar{q}_{i}}\right) \\
& \quad \times \delta\left(p_{1}+p_{2}+p_{3}-p\right) .
\end{aligned}
$$

Here the $\delta$ functions are used to guarantee the momentum conservation. The factors $\mathcal{R}_{\bar{q}_{1} q_{2}}^{(f)}$ and $\mathcal{R}_{q_{1} q_{2} q_{3}}^{(f)}$ contain Kronecker $\delta$ 's to guarantee the quark flavor conservation, e.g., if $M_{j}$ is a $D$ meson with constituent quark content $\bar{q} c, \mathcal{R}_{\bar{q}_{1} q_{2}}^{(f)}=\delta_{q_{1}, q} \delta_{q_{2}, c}$. For $\mathcal{R}_{q_{1} q_{2} q_{3}}^{(f)}$, we need to include a number $N_{\text {iter }}$ to account for the fact that there are different iterations for the flavors of the three quarks and $N_{\text {iter }}=1,3$, or 6 for $q_{1}=q_{2}=q_{3}$, $q_{1}=q_{2} \neq q_{3}$, or $q_{1} \neq q_{2} \neq q_{3}$.

The factor $C_{M_{j}}$ is the probability for $M$ to be $M_{j}$ if the quark content of $M$ is the same as $M_{j}$ and similar for $C_{B_{j}}$. In the case that only $J^{P}=0^{-}$and $1^{-}$mesons and $J^{P}=(1 / 2)^{+}$ and $(3 / 2)^{+}$baryons are considered, they are determined completely by the production ratio of vector to pseudoscalar mesons and that of $J^{P}=(1 / 2)^{+}$to $J^{P}=(3 / 2)^{+}$baryons with the same flavor content.

The remaining factor $\mathcal{R}_{M, \bar{q}_{1} q_{2}}$ now stands for the probability of a $\bar{q}_{1} q_{2}$ pair with momenta $p_{1}$ and $p_{2}$ to combine into a meson $M$ with any momentum and other quantum numbers and similarly for $\mathcal{R}_{B, q_{1} q_{2} q_{3}}$. They depend on the momenta of the (anti)quarks and their situated environments represented by $N_{q_{i}}$ and $N_{\bar{q}_{i}}$ and should be determined by the dynamics in the combination process.

In this way, we obtain the momentum distribution for a charmed meson $D_{j}$ with quark flavor content $\bar{q}_{1} c$ and that for a singly charmed baryon $B_{j}^{c}$ with $q_{1} q_{2} c$ as

$$
\begin{aligned}
f_{D_{j}}\left(p ; N_{q_{i}}, N_{\bar{q}_{i}}\right)= & \int d p_{1} d p_{2} N_{\bar{q}_{1} c} f_{\bar{q}_{1} c}^{(n)}\left(p_{1}, p_{2} ; N_{q_{i}}, N_{\bar{q}_{i}}\right) \\
& \times C_{D_{j}} \mathcal{R}_{M, \bar{q}_{1} c}\left(p_{1}, p_{2} ; N_{q_{i}}, N_{\bar{q}_{i}}\right) \\
& \times \delta\left(p_{1}+p_{2}-p\right), \\
f_{B_{j}^{c}}\left(p ; N_{q_{i}}, N_{\bar{q}_{i}}\right)= & \int d p_{1} d p_{2} d p_{3} \\
& \times N_{q_{1} q_{2} c} f_{q_{1} q_{2} c}^{(n)}\left(p_{1}, p_{2}, p_{3} ; N_{q_{i}}, N_{\bar{q}_{i}}\right) C_{B_{j}^{c}} N_{\text {iter }} \\
& \times \mathcal{R}_{B, q_{1} q_{2} c}\left(p_{1}, p_{2}, p_{3} ; N_{q_{i}}, N_{\bar{q}_{i}}\right) \\
& \times \delta\left(p_{1}+p_{2}+p_{3}-p\right) .
\end{aligned}
$$

Here, as well as in the following of this article, when talking about charmed mesons $D_{j}$ and singly charmed baryons $B_{j}^{c}, q_{1}$, $q_{2}$, and $\bar{q}_{1}$ denote $u, d$, and $s$ flavors of quarks or antiquarks. 
We emphasize that, as usual in combination models, in this article we focus the study on the charmed hadron production in the momentum space where a series of novel phenomena have been observed experimentally. The number of constraints discussed in the second paragraph of this subsection when constructing kernel functions do not include those from coordinate space. The influence from the coordinate space distribution and correlation to the momentum space will be another important and interesting aspect of future studies on combination models. See, e.g., Refs. [31,38] and the references given there.

\section{B. The momentum distribution under EVC}

It has been shown that [34] EVC seems to work well for light and strange hadron production in low- and intermediate$p_{T}$ regions. For charmed hadrons, the mass differences between the charm and the other quarks are large. It is therefore much more sensitive and more interesting to see whether the combination proceeds via EVC or other rules [9].

Under EVC, $\mathcal{R}_{M, \bar{q}_{1} q_{2}}$ and $\mathcal{R}_{B, q_{1} q_{2} q_{3}}$ take

$$
\begin{aligned}
& \mathcal{R}_{M, \bar{q}_{1} q_{2}}\left(p_{1}, p_{2} ; N_{q_{i}}, N_{\bar{q}_{i}}\right) \\
& =\mathcal{A}_{M, \bar{q}_{1} q_{2}}\left(N_{q_{i}}, N_{\bar{q}_{i}}\right) \frac{m_{q_{1}}+m_{q_{2}}}{m_{q_{1}} m_{q_{2}}} \delta\left(\frac{p_{1}}{m_{q_{1}}}-\frac{p_{2}}{m_{q_{2}}}\right), \\
& \mathcal{R}_{B, q_{1} q_{2} q_{3}}\left(p_{1}, p_{2}, p_{3} ; N_{q_{i}}, N_{\bar{q}_{i}}\right) \\
& =\mathcal{A}_{B, q_{1} q_{2} q_{3}}\left(N_{q_{i}}, N_{\bar{q}_{i}}\right) \frac{m_{q_{1}}+m_{q_{2}}+m_{q_{3}}}{m_{q_{1}} m_{q_{2}} m_{q_{3}}} \delta\left(\frac{p_{1}}{m_{q_{1}}}-\frac{p_{2}}{m_{q_{2}}}\right) \\
& \quad \times \delta\left(\frac{p_{1}}{m_{q_{1}}}-\frac{p_{3}}{m_{q_{3}}}\right),
\end{aligned}
$$

where $m_{q_{i}}$ is the constituent quark mass and is taken as $m_{u}=m_{d}=0.33 \mathrm{GeV}, m_{s}=0.5 \mathrm{GeV}$, and $m_{c}=1.5 \mathrm{GeV}$ in the numerical calculations in this article; and $\mathcal{A}_{M, \bar{q}_{1} q_{2}}$ and $\mathcal{A}_{B, q_{1} q_{2} q_{3}}$ are factors denoting the meson-baryon production competition as well as guaranteeing the unitarity. They depend on the numbers of different flavor quarks and antiquarks in the considered system. The mass term is introduced for normalization, so that the original kernel functions in Eqs. (1) and (2) take the following forms:

$$
\begin{aligned}
& \mathcal{R}_{M_{j}, \bar{q}_{1} q_{2}}\left(p_{1}, p_{2} ; N_{q_{i}}, N_{\bar{q}_{i}}\right) \\
& =C_{M_{j}} \mathcal{R}_{\bar{q}_{1} q_{2}}^{(f)} \mathcal{A}_{M, \bar{q}_{1} q_{2}}\left(N_{q_{i}}, N_{\bar{q}_{i}}\right) \\
& \quad \times \delta\left(p_{1}-x_{q_{1} q_{2}}^{q_{1}} p\right) \delta\left(p_{2}-x_{q_{1} q_{2}}^{q_{2}} p\right), \\
& \mathcal{R}_{B_{j}, q_{1} q_{2} q_{3}}\left(p_{1}, p_{2}, p_{3} ; N_{q_{i}}, N_{\bar{q}_{i}}\right) \\
& =C_{B_{j}} \mathcal{R}_{q_{1} q_{2} q_{3}}^{(f)} \mathcal{A}_{B, q_{1} q_{2} q_{3}}\left(N_{q_{i}}, N_{\bar{q}_{i}}\right) \delta\left(p_{1}-x_{q_{1} q_{2} q_{3}}^{q_{1}} p\right) \\
& \quad \times \delta\left(p_{2}-x_{q_{1} q_{2} q_{3}}^{q_{2}} p\right) \delta\left(p_{3}-x_{q_{1} q_{2} q_{3}}^{q_{3}} p\right),
\end{aligned}
$$

where $x_{q_{1} q_{2}}^{q_{i}}=m_{q_{i}} /\left(m_{q_{1}}+m_{q_{2}}\right)$ and $x_{q_{1} q_{2} q_{3}}^{q_{i}}=m_{q_{i}} /\left(m_{q_{1}}+\right.$ $\left.m_{q_{2}}+m_{q_{3}}\right)$ are the fractions of the momentum of the produced hadron carried by $q_{i}$.

We note that because the purpose of this article is to study the momentum distributions of charmed hadrons, we use the momentum as variable and express the kernel function determined by EVC in the form of Eqs. (7) and (8). It can also be expressed in other forms in terms of other variables depending on the properties that one wishes to describe. For example, to study the rapidity distribution, we use the rapidity $y$ as variable and the $\delta$ function in Eqs. (7) and (8) should be replaced by $\delta\left(y_{1}-y_{i}\right)$ multiplied by the kinematic factor obtained from the Jacobi due to the variable change. It is also interesting to see that in this case, EVC is just similar to the so-called "near correlation in rapidity" discussed in quark combination models some time ago [39].

Substituting Eqs. (7) and (8) into Eqs. (5) and (6) and carrying out the integration over the quark momenta, we obtain

$$
\begin{aligned}
f_{D_{j}}(p)= & C_{D_{j}} \mathcal{A}_{M, \bar{q}_{1} c}\left(N_{q_{i}}, N_{\bar{q}_{i}}\right) N_{\bar{q}_{1} c} f_{\bar{q}_{1} c}^{(n)}\left(x_{q_{1} c}^{q_{1}} p, x_{q_{1} c}^{c} p\right), \\
f_{B_{j}^{c}}(p)= & C_{B_{j}^{c}} \mathcal{A}_{B, q_{1} q_{2} c}\left(N_{q_{i}}, N_{\bar{q}_{i}}\right) N_{q_{1} q_{2} c} N_{\mathrm{iter}} \\
& \times f_{q_{1} q_{2} c}^{(n)}\left(x_{q_{1} q_{2} c}^{q_{1}} p, x_{q_{1} q_{2} c}^{q_{2}} p, x_{q_{1} q_{2} c}^{c} p\right) .
\end{aligned}
$$

Here and from now on in this article, we suppress the arguments $N_{q_{i}}$ and $N_{\bar{q}_{i}}$ in the momentum distribution functions for explicitness.

The factor $\mathcal{A}_{M, \bar{q}_{1} c}\left(N_{q_{i}}, N_{\bar{q}_{i}}\right)$ is the probability for a charm quark to capture a specific antiquark $\bar{q}_{1}$ to form a meson in the bulk quark-antiquark system; it should be inversely proportional to the total number of quarks and antiquarks $N_{q}+N_{\bar{q}}$. Similarly, $\mathcal{A}_{B, q_{1} q_{2} c}\left(N_{q_{i}}, N_{\bar{q}_{i}}\right)$ should be proportional to $1 /\left(N_{q}+N_{\bar{q}}\right)^{2}$. Therefore, one can write

$$
\begin{gathered}
\mathcal{A}_{M, \bar{q}_{1} c}\left(N_{q_{i}}, N_{\bar{q}_{i}}\right)=\mathcal{A}_{M} /\left(N_{q}+N_{\bar{q}}\right), \\
\mathcal{A}_{B, q_{1} q_{2} c}\left(N_{q_{i}}, N_{\bar{q}_{i}}\right)=\mathcal{A}_{B} /\left(N_{q}+N_{\bar{q}}\right)^{2},
\end{gathered}
$$

where $\mathcal{A}_{M}$ and $\mathcal{A}_{B}$ are proportionality coefficients and they closely relate to the unitarity and the meson-baryon production competition. For a given quark-antiquark system, $\mathcal{A}_{M}$ and $\mathcal{A}_{B}$ should be universal for all different $D$ mesons and singly charmed baryons due to the quark flavor blindness of the strong interaction. Substituting Eqs. (13) and (14) into Eqs. (11) and (12), respectively, we have

$$
\begin{aligned}
& f_{D_{j}}(p)=N_{c} \mathcal{A}_{M} C_{D_{j}} \lambda_{\bar{q}_{1}} f_{\bar{q}_{1} c}^{(n)}\left(x_{q_{1} c}^{q_{1}} p, x_{q_{1} c}^{c} p\right), \\
& f_{B_{j}^{c}}(p)=N_{c} \mathcal{A}_{B} C_{B_{j}^{c}} \lambda_{q_{1} q_{2}} N_{\text {iter }} f_{q_{1} q_{2} c}^{(n)}\left(x_{q_{1} q_{2} c}^{q_{1}} p, x_{q_{1} q_{2} c}^{q_{2}} p, x_{q_{1} q_{2} c}^{c} p\right),
\end{aligned}
$$

where $\lambda_{\bar{q}_{1}} \equiv N_{\bar{q}_{1}} /\left(N_{q}+N_{\bar{q}}\right)$ and $\lambda_{q_{1} q_{2}} \equiv N_{q_{1} q_{2}} /\left(N_{q}+N_{\bar{q}}\right)^{2}$. If we consider a quark-antiquark system in the midrapidity region at high energies so that the influence of net quarks from the colliding nuclei can be neglected, the ratio $\lambda_{\bar{q}_{1}}$ and $\lambda_{q_{1} q_{2}}$ are both determined completely by the strangeness suppression factor $\lambda_{s}$.

If we neglect correlations of momentum distributions of quarks and/or antiquarks of different flavors in the system, i.e., we take

$$
\begin{gathered}
f_{\bar{q}_{1} q_{2}}^{(n)}\left(p_{1}, p_{2}\right)=f_{\bar{q}_{1}}^{(n)}\left(p_{1}\right) f_{q_{2}}^{(n)}\left(p_{2}\right), \\
f_{q_{1} q_{2} q_{3}}^{(n)}\left(p_{1}, p_{2}, p_{3}\right)=f_{q_{1}}^{(n)}\left(p_{1}\right) f_{q_{2}}^{(n)}\left(p_{2}\right) f_{q_{3}}^{(n)}\left(p_{3}\right) .
\end{gathered}
$$

In this case, we obtain

$$
f_{D_{j}}(p)=N_{c} \mathcal{A}_{M} C_{D_{j}} \lambda_{\bar{q}_{1}} f_{\bar{q}_{1}}^{(n)}\left(x_{q_{1} c}^{q_{1}} p\right) f_{c}^{(n)}\left(x_{q_{1} c}^{c} p\right),
$$




$$
\begin{aligned}
f_{B_{j}^{c}}(p)= & N_{c} \mathcal{A}_{B} C_{B_{j}^{c}} \lambda_{q_{1} q_{2}} N_{\text {iter }} \\
& \times f_{q_{1}}^{(n)}\left(x_{q_{1} q_{2} c}^{q_{1}} p\right) f_{q_{2}}^{(n)}\left(x_{q_{1} q_{2} c}^{q_{2}} p\right) f_{c}^{(n)}\left(x_{q_{1} q_{2} c}^{c} p\right) .
\end{aligned}
$$

By using Eqs. (19) and (20), we can calculate momentum distributions and ratios for the production of different charmed hadrons. The factors $\mathcal{A}_{M}$ and $\mathcal{A}_{B}$ are determined by charm quark number conservation in the combination process and the charmed baryon-to-meson production ratio $N_{B^{c}} / N_{D}$. Here $N_{D}$ and $N_{B^{c}}$ are the total number of all the produced $D$ mesons and that of singly charmed baryons. For the charm quark number conservation, we need in principle to consider the production of hadrons besides singly charmed ones such as charmonia, doubly and triply charmed baryons, and even exotic states. However, the production rates for them are very small and they exhaust about less than $5 \%$ of total charm quarks [40]. In the numerical calculations in the following of this article, we neglect them so the charm quark number conservation just takes $N_{D}+N_{B^{c}} \approx N_{c}$. In this approximation, $\mathcal{A}_{M}$ and $\mathcal{A}_{B}$ are just determined by the ratio $R_{B / M}^{(c)} \equiv N_{B^{c}} / N_{D}$ that is taken as a parameter fixed by the experimental data of one yield ratio such as $\Lambda_{c}^{+} / D^{0}$ in the calculations.

\section{Decay contributions}

To compare with the experimental data, we need to include strong and electromagnetic decay contributions from shortlived charmed resonances [41]. In our case, we need only to consider decays of $D^{*}$ mesons, $J^{P}=(3 / 2)^{+}$singly charmed baryons, and $\Sigma_{c}$ baryons. They all decay into a $D$ meson or a $J^{P}=(1 / 2)^{+}$singly charmed baryon with a light particle such as a pion or a photon. In such a decay process, the momentum of the light daughter particle is so small that can be neglected compared to that of the heavy daughter charmed hadron. We can approximately take the momentum of the daughter charmed hadron equal to that of the mother charmed hadron. In this approximation, we have, e.g., for $D^{0}$ mesons,

$$
\begin{aligned}
f_{D^{0}}^{(\mathrm{fin})}(p) \approx & f_{D^{0}}(p)+\operatorname{Br}\left(D^{* 0} \rightarrow D^{0}\right) f_{D^{* 0}}(p) \\
& +\operatorname{Br}\left(D^{*+} \rightarrow D^{0}\right) f_{D^{*+}}(p)
\end{aligned}
$$

where Br's are the decay branch ratios and $100 \%$ and $67.7 \%$ for $D^{* 0}$ and $D^{*+}$, respectively [41]. It follows from Eq. (19) that

$$
f_{D^{* 0}}(p)=f_{D^{*+}}(p)=R_{V / P} f_{D^{0}}(p)
$$

where $R_{V / P}$ is the ratio of the production rate of vector to pseudoscalar mesons with the same quark contents and is taken as $R_{V / P}=1.5$ [35,42]. In this way, we obtain the result for $f_{D^{0}}^{(\mathrm{fin})}(p)$ and similar results for other charmed mesons as follows:

$$
\begin{gathered}
f_{D^{0}}^{(\mathrm{fin})}(p) \approx 3.516 f_{D^{0}}(p), \\
f_{D^{+}}^{(\mathrm{fin})}(p) \approx 1.485 f_{D^{+}}(p), \\
f_{D_{s}^{+}}^{(\mathrm{fin})}(p) \approx 2.5 f_{D_{s}^{+}}(p) .
\end{gathered}
$$

Similarly, we take the $J^{P}=(1 / 2)^{+}$to $J^{P}=(3 / 2)^{+}$singly charmed baryon ratio as 2 [35] and obtain the following:

$$
\begin{gathered}
f_{\Lambda_{c}^{+}}^{(\mathrm{fin})}(p) \approx 5 f_{\Lambda_{c}^{+}}(p), \\
f_{\Sigma_{c}^{0}}^{(\mathrm{fin})}(p) \approx f_{\Sigma_{c}^{0}}(p), \\
f_{\Sigma_{c}^{+}}^{(\mathrm{fin})}(p) \approx f_{\Sigma_{c}^{+}}(p), \\
f_{\Sigma_{c}^{++}}^{(\mathrm{fin})}(p) \approx f_{\Sigma_{c}^{++}}(p), \\
f_{\Xi_{c}^{0}}^{(\mathrm{fin})}(p) \approx 2.5 f_{\Xi_{c}^{0}}(p), \\
f_{\Xi_{c}^{+}}^{(\mathrm{fin})}(p) \approx 2.5 f_{\Xi_{c}^{+}}(p), \\
f_{\Omega_{c}^{0}}^{(\mathrm{fin})}(p) \approx 1.5 f_{\Omega_{c}^{0}}(p) .
\end{gathered}
$$

From these results, we see clearly that contributions from resonance decays are important for most of the charmed hadrons. For example, for $\Lambda_{c}^{+}$baryons, about $80 \%$ are from decay contributions and only about $20 \%$ are directly produced ones.

\section{Ratios of different hadrons}

We consider the production of charmed hadrons at midrapidity $y=0$ and apply Eqs. (19) and (20) to obtain the $p_{T}$ dependence. From them, we calculate ratios of different charmed hadrons. In this case, the numbers of different flavor quarks are just replaced by the number densities $d N_{q_{i}} / d y$ at $y=0$.

We first consider yield ratios of strange to nonstrange hadrons in the charm sector. We use the results given by Eqs. (23)-(32) to build the corresponding ratios and obtain the following:

$$
\begin{aligned}
& \frac{D_{s}^{+}}{D^{0}}=0.711 \lambda_{s} \frac{f_{\bar{s}}^{(n)}\left(x_{\mathrm{sc}}^{s} p_{T}\right) f_{c}^{(n)}\left(x_{\mathrm{sc}}^{c} p_{T}\right)}{f_{\bar{d}}^{(n)}\left(x_{\mathrm{dc}}^{d} p_{T}\right) f_{c}^{(n)}\left(x_{\mathrm{dc}}^{c} p_{T}\right)}, \\
& \frac{\Xi_{c}^{+}}{\Lambda_{c}^{+}}=0.5 \lambda_{s} \frac{f_{d}^{(n)}\left(x_{\mathrm{dsc}}^{d} p_{T}\right) f_{s}^{(n)}\left(x_{\mathrm{dsc}}^{s} p_{T}\right) f_{c}^{(n)}\left(x_{\mathrm{dsc}}^{c} p_{T}\right)}{\left[f_{d}^{(n)}\left(x_{\mathrm{ddc}}^{d} p_{T}\right)\right]^{2} f_{c}^{(n)}\left(x_{\mathrm{ddc}}^{c} p_{T}\right)}, \\
& \frac{\Omega_{c}^{0}}{\Xi_{c}^{0}}=0.5 \lambda_{s} \frac{\left[f_{s}^{(n)}\left(x_{\mathrm{ssc}}^{s} p_{T}\right)\right]^{2} f_{c}^{(n)}\left(x_{\mathrm{ssc}}^{c} p_{T}\right)}{f_{d}^{(n)}\left(x_{\mathrm{dsc}}^{d} p_{T}\right) f_{s}^{(n)}\left(x_{\mathrm{dsc}}^{s} p_{T}\right) f_{c}^{(n)}\left(x_{\mathrm{dsc}}^{c} p_{T}\right)}, \\
& \frac{\Omega_{c}^{0}}{\Lambda_{c}^{+}}=0.25 \lambda_{s}^{2} \frac{\left[f_{s}^{(n)}\left(x_{\mathrm{ssc}}^{s} p_{T}\right)\right]^{2} f_{c}^{(n)}\left(x_{\mathrm{ssc}}^{c} p_{T}\right)}{\left[f_{d}^{(n)}\left(x_{\mathrm{ddc}}^{d} p_{T}\right)\right]^{2} f_{c}^{(n)}\left(x_{\mathrm{ddc}}^{c} p_{T}\right)} .
\end{aligned}
$$

We can use them to calculate these yield ratios numerically. Here we see clearly that these ratios depend not only on the strangeness suppression factor $\lambda_{s}$ but also on the ratios of $p_{T}$ distributions of different flavors of (anti)quarks.

To see the qualitative features more explicitly, we note that, because $m_{c}$ is much larger than $m_{d}$ and $m_{s}$, it dominates the sum of $m_{c}$ with other quark masses. We have that $x_{q_{1} q_{1} c}^{q_{1}} \approx x_{q_{1} q_{2} c}^{q_{1}} \approx x_{q_{1} c}^{q_{1}}$ and $x_{q_{1} q_{2} c}^{c} \approx x_{q_{1} c}^{c} \approx x_{q_{2} c}^{c}$, and the latter should be much larger than the former. We take, in a rough 
approximation, the former as the same, and rewrite Eqs. (33)(36) in the following forms:

$$
\begin{aligned}
& \frac{D_{s}^{+}}{D^{0}}=0.711 \lambda_{s} \frac{f_{\bar{s}}^{(n)}\left(x_{\mathrm{sc}}^{s} p_{T}\right)}{f_{\bar{d}}^{(n)}\left(x_{\mathrm{dc}}^{d} p_{T}\right)} \frac{f_{c}^{(n)}\left(x_{\mathrm{sc}}^{c} p_{T}\right)}{f_{c}^{(n)}\left(x_{\mathrm{dc}}^{c} p_{T}\right)}, \\
& \frac{\Xi_{c}^{+}}{\Lambda_{c}^{+}} \approx 0.5 \lambda_{s} \frac{f_{s}^{(n)}\left(x_{\mathrm{dsc}}^{s} p_{T}\right)}{f_{d}^{(n)}\left(x_{\mathrm{ddc}}^{d} p_{T}\right)} \frac{f_{c}^{(n)}\left(x_{\mathrm{dsc}}^{c} p_{T}\right)}{f_{c}^{(n)}\left(x_{\mathrm{ddc}}^{c} p_{T}\right)}, \\
& \frac{\Omega_{c}^{0}}{\Xi_{c}^{0}} \approx 0.5 \lambda_{s} \frac{f_{s}^{(n)}\left(x_{\mathrm{ssc}}^{s} p_{T}\right)}{f_{d}^{(n)}\left(x_{\mathrm{dsc}}^{d} p_{T}\right)} \frac{f_{c}^{(n)}\left(x_{\mathrm{ssc}}^{c} p_{T}\right)}{f_{c}^{(n)}\left(x_{\mathrm{dsc}}^{c} p_{T}\right)}, \\
& \frac{\Omega_{c}^{0}}{\Lambda_{c}^{+}}=0.25 \lambda_{s}^{2}\left[\frac{f_{s}^{(n)}\left(x_{\mathrm{ssc}}^{s} p_{T}\right)}{f_{d}^{(n)}\left(x_{\mathrm{ddc}}^{d} p_{T}\right)}\right]^{2} \frac{f_{c}^{(n)}\left(x_{\mathrm{ssc}}^{c} p_{T}\right)}{f_{c}^{(n)}\left(x_{\mathrm{ddc}}^{c} p_{T}\right)} .
\end{aligned}
$$

Here, we see clearly that, besides $\lambda_{s}$ (or $\lambda_{s}^{2}$ ), these ratios are proportional to the ratio of $s$ - to $d$-quark spectrum (or squared) and the ratio of $c$-quark spectrum at slightly different $p_{T}$ values. Because the values of $x$ involved here are all quite small for $d$ and $s$ quarks, they should be sensitive to the $d$ - and $s$-quark $p_{T}$ spectra in the relatively low- $p_{T}$ regions. The observed enhancement of strange to nonstrange charmed hadron ratios does not necessarily come from the enhancement of $\lambda_{s}$ but can also come from the influence of the $p_{T}$ spectra of quarks.

Similarly, for baryon-to-meson ratios, we obtain from Eqs. (23)-(32) that

$$
\begin{aligned}
& \frac{\Lambda_{c}^{+}}{D^{0}}=\frac{4.267}{2+\lambda_{s}} \frac{\mathcal{A}_{B}}{\mathcal{A}_{M}} \frac{\left[f_{d}^{(n)}\left(x_{\mathrm{ddc}}^{d} p_{T}\right)\right]^{2} f_{c}^{(n)}\left(x_{\mathrm{ddc}}^{c} p_{T}\right)}{f_{\bar{d}}^{(n)}\left(x_{\mathrm{dc}}^{d} p_{T}\right) f_{c}^{(n)}\left(x_{\mathrm{dc}}^{c} p_{T}\right)}, \\
& \frac{\Sigma_{c}^{0}}{D^{0}}=\frac{0.711}{2+\lambda_{s}} \frac{\mathcal{A}_{B}}{\mathcal{A}_{M}} \frac{\left[f_{d}^{(n)}\left(x_{\mathrm{ddc}}^{d} p_{T}\right)\right]^{2} f_{c}^{(n)}\left(x_{\mathrm{ddc}}^{c} p_{T}\right)}{f_{\bar{d}}^{(n)}\left(x_{\mathrm{dc}}^{d} p_{T}\right) f_{c}^{(n)}\left(x_{\mathrm{dc}}^{c} p_{T}\right)}, \\
& \frac{\Sigma_{c}^{++}}{D^{+}}=\frac{1.684}{2+\lambda_{s}} \frac{\mathcal{A}_{B}}{\mathcal{A}_{M}} \frac{\left[f_{d}^{(n)}\left(x_{\mathrm{ddc}}^{d} p_{T}\right)\right]^{2} f_{c}^{(n)}\left(x_{\mathrm{ddc}}^{c} p_{T}\right)}{f_{\bar{d}}^{(n)}\left(x_{\mathrm{dc}}^{d} p_{T}\right) f_{c}^{(n)}\left(x_{\mathrm{dc}}^{c} p_{T}\right)}, \\
& \frac{\Xi_{c}^{0}}{D^{0}}=\frac{2.133 \lambda_{s}}{2+\lambda_{s}} \frac{\mathcal{A}_{B}}{\mathcal{A}_{M}} \frac{f_{d}^{(n)}\left(x_{\mathrm{dsc}}^{d} p_{T}\right) f_{s}^{(n)}\left(x_{\mathrm{dsc}}^{s} p_{T}\right) f_{c}^{(n)}\left(x_{\mathrm{dsc}}^{c} p_{T}\right)}{f_{\bar{d}}^{(n)}\left(x_{\mathrm{dc}}^{d} p_{T}\right) f_{c}^{(n)}\left(x_{\mathrm{dc}}^{c} p_{T}\right)}, \\
& \frac{\Xi_{c}^{+}}{D_{s}^{+}}=\frac{3}{2+\lambda_{s}} \frac{\mathcal{A}_{B}}{\mathcal{A}_{M}} \frac{f_{d}^{(n)}\left(x_{\mathrm{dsc}}^{d} p_{T}\right) f_{s}^{(n)}\left(x_{\mathrm{dsc}}^{s} p_{T}\right) f_{c}^{(n)}\left(x_{\mathrm{dsc}}^{c} p_{T}\right)}{f_{\bar{s}}^{(n)}\left(x_{\mathrm{sc}}^{s} p_{T}\right) f_{c}^{(n)}\left(x_{\mathrm{sc}}^{c} p_{T}\right)}, \\
& \frac{\Omega_{c}^{0}}{D^{0}}=\frac{1.067 \lambda_{s}^{2}}{2+\lambda_{s}} \frac{\mathcal{A}_{B}}{\mathcal{A}_{M}} \frac{\left[f_{s}^{(n)}\left(x_{\mathrm{ssc}}^{s} p_{T}\right)\right]^{2} f_{c}^{(n)}\left(x_{\mathrm{ssc}}^{c} p_{T}\right)}{f_{\bar{d}}^{(n)}\left(x_{\mathrm{dc}}^{d} p_{T}\right) f_{c}^{(n)}\left(x_{\mathrm{dc}}^{c} p_{T}\right)}, \\
& \frac{\Omega_{c}^{0}}{D_{s}^{+}}=\frac{1.5 \lambda_{s}}{2+\lambda_{s}} \frac{\mathcal{A}_{B}}{\mathcal{A}_{M}} \frac{\left[f_{s}^{(n)}\left(x_{\mathrm{ssc}}^{s} p_{T}\right)\right]^{2} f_{c}^{(n)}\left(x_{\mathrm{ssc}}^{c} p_{T}\right)}{f_{\bar{s}}^{(n)}\left(x_{\mathrm{sc}}^{s} p_{T}\right) f_{c}^{(n)}\left(x_{\mathrm{sc}}^{c} p_{T}\right)} \text {. }
\end{aligned}
$$

We see again that, besides the strangeness suppression factor $\lambda_{s}$ and $\mathcal{A}_{B} / \mathcal{A}_{M}$ (determined by the charmed baryon-tomeson ratio $\left.R_{B / M}^{(c)}\right)$, these ratios depend also on the $p_{T}$ spectra of quarks. Similarly, in the rough estimation with $x_{q_{1} q_{1} c}^{q_{1}} \approx$ $x_{q_{1} q_{2} c}^{q_{1}} \approx x_{q_{1} c}^{q_{1}}$, we can rewrite them as

$$
\begin{aligned}
& \frac{\Lambda_{c}^{+}}{D^{0}} \approx \frac{4.267}{2+\lambda_{s}} \frac{\mathcal{A}_{B}}{\mathcal{A}_{M}} f_{d}^{(n)}\left(x_{\mathrm{ddc}}^{d} p_{T}\right) \frac{f_{c}^{(n)}\left(x_{\mathrm{ddc}}^{c} p_{T}\right)}{f_{c}^{(n)}\left(x_{\mathrm{dc}}^{c} p_{T}\right)}, \\
& \frac{\Sigma_{c}^{0}}{D^{0}} \approx \frac{0.711}{2+\lambda_{s}} \frac{\mathcal{A}_{B}}{\mathcal{A}_{M}} f_{d}^{(n)}\left(x_{\mathrm{ddc}}^{d} p_{T}\right) \frac{f_{c}^{(n)}\left(x_{\mathrm{ddc}}^{c} p_{T}\right)}{f_{c}^{(n)}\left(x_{\mathrm{dc}}^{c} p_{T}\right)}, \\
& \frac{\Sigma_{c}^{++}}{D^{+}} \approx \frac{1.684}{2+\lambda_{s}} \frac{\mathcal{A}_{B}}{\mathcal{A}_{M}} f_{d}^{(n)}\left(x_{\mathrm{ddc}}^{d} p_{T}\right) \frac{f_{c}^{(n)}\left(x_{\mathrm{ddc}}^{c} p_{T}\right)}{f_{c}^{(n)}\left(x_{\mathrm{dc}}^{c} p_{T}\right)}, \\
& \frac{\Xi_{c}^{0}}{D^{0}} \approx \frac{2.133 \lambda_{s}}{2+\lambda_{s}} \frac{\mathcal{A}_{B}}{\mathcal{A}_{M}} f_{s}^{(n)}\left(x_{\mathrm{dsc}}^{s} p_{T}\right) \frac{f_{c}^{(n)}\left(x_{\mathrm{dsc}}^{c} p_{T}\right)}{f_{c}^{(n)}\left(x_{\mathrm{dc}}^{c} p_{T}\right)}, \\
& \frac{\Xi_{c}^{+}}{D_{s}^{+}} \approx \frac{3}{2+\lambda_{s}} \frac{\mathcal{A}_{B}}{\mathcal{A}_{M}} f_{d}^{(n)}\left(x_{\mathrm{dsc}}^{d} p_{T}\right) \frac{f_{c}^{(n)}\left(x_{\mathrm{dsc}}^{c} p_{T}\right)}{f_{c}^{(n)}\left(x_{\mathrm{sc}}^{c} p_{T}\right)}, \\
& \quad \frac{\Omega_{c}^{0}}{D^{0}}=\frac{1.067 \lambda_{s}^{2}}{2+\lambda_{s}} \frac{\mathcal{A}_{B}}{\mathcal{A}_{M}} f_{s}^{(n)}\left(x_{\mathrm{ssc}}^{s} p_{T}\right) \\
& \frac{f_{s}^{(n)}\left(x_{\mathrm{sc}}^{s} p_{T}\right)}{f_{\bar{d}}^{(n)}\left(x_{\mathrm{dc}}^{c} p_{T}\right)} \frac{f_{c}^{(n)}\left(x_{\mathrm{ssc}}^{c} p_{T}\right)}{f_{c}^{(n)}\left(x_{\mathrm{dc}}^{c} p_{T}\right)}, \\
& \Omega_{c}^{0} \frac{1.5 \lambda_{s}}{2+\lambda_{s}} \frac{\mathcal{A}_{B}}{\mathcal{A}_{M}} f_{s}^{(n)}\left(x_{\mathrm{ssc}}^{s} p_{T}\right) \frac{f_{c}^{(n)}\left(x_{\mathrm{ssc}}^{c} p_{T}\right)}{f_{c}^{(n)}\left(x_{\mathrm{sc}}^{c} p_{T}\right)} .
\end{aligned}
$$

Here we see clearly that these ratios, besides $\lambda_{s}$ and $\mathcal{A}_{B} / \mathcal{A}_{M}$, should be sensitive to the $p_{T}$ spectrum of $d$ or $s$ quarks in the relatively small- $p_{T}$ region. Because the $p_{T}$ distribution $f_{q_{i}}^{(n)}\left(p_{T}\right)$ of $d$ or $s$ quarks in such $p_{T}$ regions typically exhibits "rise-peak-fall" behaviors, we expect that these charmed baryon-to-meson ratios should have similar risepeak-fall behaviors. We also expect that these ratios should have $p_{T}$ dependencies much stronger than those of strange to nonstrange ratios given by Eqs. (33)-(36) because the latter depends only on ratios of $p_{T}$ spectra of quarks but the former depends on the spectrum itself. We would like to emphasize that Eqs. (41)-(47) are characteristic results in the equal-velocity quark combination (EVC). They can be directly used to test the combination hadronization mechanism of charm quarks and the validity of the EVC. They may also provide special ways to probe the properties of the QGP due to their close relationships to low- $p_{T}$ spectra of $d$ and $s$ quarks.

\section{APPLICATIONS IN Au-Au COLLISIONS AT RHIC}

In this section, we apply the deduced results in Sec. II to calculate the charmed hadron production at midrapidity in $\mathrm{Au}-\mathrm{Au}$ collisions at $\sqrt{s_{\mathrm{NN}}}=200 \mathrm{GeV}$. We first present the normalized $p_{T}$ spectra of quarks and other related parameters. We then give numerical results of hadron yield ratios. We finally present predictions for $p_{T}$ spectra and $p_{T}$-integrated yield densities of different charmed hadrons.

\section{A. The normalized $p_{T}$ spectra of quarks}

In the midrapidity region of $\mathrm{Au}-\mathrm{Au}$ collisions at high energies, we can neglect net quark contributions and take isospin symmetric quark distributions. In this case, we only need the three parameters $\lambda_{s}, R_{B / M}^{(c)}$, and $d N_{c} / d y$ and normalized $p_{T}$ spectra of $d, s$, and $c$ quarks as inputs. In QGP in heavy-ion 
TABLE I. $\lambda_{s}$ and parameters for quark distributions in different centralities in Au-Au collisions at $\sqrt{s_{\mathrm{NN}}}=200 \mathrm{GeV}$.

\begin{tabular}{lccccc}
\hline \hline Centrality & $0-10 \%$ & $10-20 \%$ & $20-40 \%$ & $40-60 \%$ & $60-80 \%$ \\
\hline$\lambda_{s}$ & 0.49 & 0.46 & 0.45 & 0.45 & 0.44 \\
$T_{d}(\mathrm{GeV})$ & 0.27 & 0.26 & 0.25 & 0.24 & 0.23 \\
$T_{s}(\mathrm{GeV})$ & 0.34 & 0.34 & 0.34 & 0.33 & 0.32 \\
$\alpha_{d}$ & 0.65 & 0.65 & 0.65 & 0.62 & 0.58 \\
$\alpha_{s}$ & 0.65 & 0.65 & 0.65 & 0.62 & 0.58 \\
$\alpha_{c}\left(\mathrm{GeV}^{-0.5}\right)$ & 11 & 10 & 9 & 7 & 6 \\
$T_{c}(\mathrm{GeV})$ & 0.45 & 0.45 & 0.45 & 0.45 & 0.44 \\
$\beta_{c}$ & 3.65 & 3.45 & 3.20 & 2.85 & 2.80 \\
\hline \hline
\end{tabular}

collisions, $\lambda_{s}$ takes values in the range $0.4-0.6$ [37]. Here, we use the yield ratio of antibaryons, such as $\bar{\Lambda}$ to $\bar{p}[37,43,44]$, to fix the value of $\lambda_{s}$, and the results in different centralities are shown in Table I. The $R_{B / M}^{(c)}$ and $d N_{c} / d y$ are given whenever they are needed.

For $f_{d}^{(n)}\left(p_{T}\right)$ and $f_{s}^{(n)}\left(p_{T}\right)$, we take the modified-thermal pattern,

$$
f_{q_{i}}^{(n)}\left(p_{T}\right) \propto p_{T}^{\alpha_{q_{i}}} \exp \left(-\sqrt{p_{T}^{2}+m_{q_{i}}^{2}} / T_{q_{i}}\right)
$$

and extract the parameters $T_{q_{i}}$ and $\alpha_{q_{i}}$ from data [44,45] on the $p_{T}$ spectra of $\Xi^{-}$baryons and $\phi$ mesons under EVC. The obtained results are given in Table I. We also plot $f_{d}^{(n)}\left(p_{T}\right)$ and $f_{s}^{(n)}\left(p_{T}\right)$ in Figs. 1(a) and 1(b).
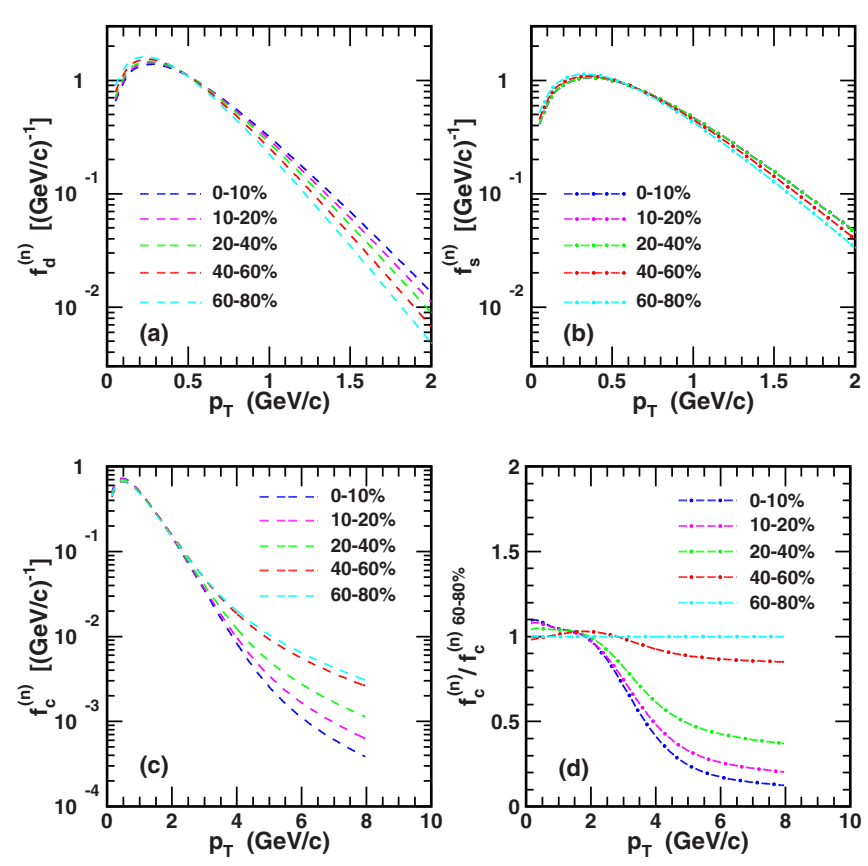

FIG. 1. Normalized $p_{T}$ spectra of (a) down, (b) strange, and (c) charm quarks in different centralities in $\mathrm{Au}-\mathrm{Au}$ collisions at $\sqrt{s_{\mathrm{NN}}}=200 \mathrm{GeV}$. In panel (d), we have ratios of $p_{T}$ distributions of charm quarks in different centralities to the $p_{T}$ distribution in the $60-80 \%$ centrality.
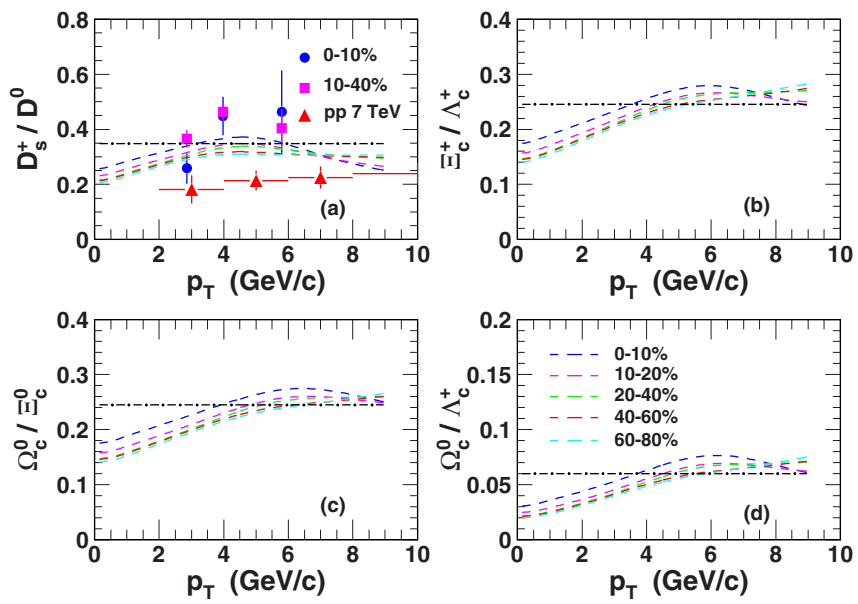

FIG. 2. Ratios of strange to nonstrange charmed hadrons (a) $D_{s}^{+} / D^{0}$, (b) $\Xi_{c}^{+} / \Lambda_{c}^{+}$, (c) $\Omega_{c}^{0} / \Xi_{c}^{0}$, and (d) $\Omega_{c}^{0} / \Lambda_{c}^{+}$, as functions of $p_{T}$ in Au-Au collisions at $\sqrt{s_{\mathrm{NN}}}=200 \mathrm{GeV}$. The solid circles and squares are data that are taken from Ref. [15]. The solid triangles denoted $p p$ reaction data at $\mathrm{LHC}$ [47] are also plotted for comparison. The dashed-dotted line in each panel represents the constant $0.711 \lambda_{s}$, $0.5 \lambda_{s}, 0.5 \lambda_{s}$, or $0.25 \lambda_{s}^{2}$ at $\lambda_{s}=0.49$, respectively. Dashed curves are the numerical results for different centralities in $\mathrm{Au}-\mathrm{Au}$ collisions.

For $c$ quarks, we adopt the hybrid pattern [46], i.e.,

$$
\begin{aligned}
f_{c}^{(n)}\left(p_{T}\right) \propto & \alpha_{c} p_{T} \exp \left(-\sqrt{p_{T}^{2}+m_{c}^{2}} / T_{c}\right) \\
& +\sqrt{p_{T}}\left[1.0+\left(\sqrt{p_{T}^{2}+m_{c}^{2}}-m_{c}\right) / 0.6\right]^{-\beta_{c}},
\end{aligned}
$$

based on the results after the propagation of charm quarks in the QGP medium in a Boltzmann transport approach [46]. The parameters $\alpha_{c}, T_{c}$, and $\beta_{c}$ are fixed using the data on the $p_{T}$ distribution of $D^{0}$ [12] and are given in Table I. We also plot in Fig. 1(c) $f_{c}^{(n)}\left(p_{T}\right)$ in different centralities in $\mathrm{Au}-\mathrm{Au}$ collisions at $\sqrt{s_{\mathrm{NN}}}=200 \mathrm{GeV}$. We see that there is a stronger suppression in more central collisions, especially in the region $4 \mathrm{GeV}<p_{T}<8 \mathrm{GeV}$. Shown in Fig. 1(d) are the ratios of these distributions in different centralities to that in the $60-80 \%$ centrality. We see behavior very similar to that of the nuclear modification factor $R_{\mathrm{CP}}$ of $D^{0}$ mesons measured in Ref. [12]. In low $p_{T}<2 \mathrm{GeV}, p_{T}$ distributions of charm quarks are almost the same for different centralities.

Comparing the results in Fig. 1(c) to those given by Figs. 1(a) and 1(b), we see that the $p_{T}$ dependence of $f_{c}^{(n)}\left(p_{T}\right)$ is much stronger than that of $f_{d}^{(n)}\left(p_{T}\right)$ or $f_{s}^{(n)}\left(p_{T}\right)$. We therefore expect that $f_{c}^{(n)}\left(p_{T}\right)$ should have large influences on both the $p_{T}$ distributions of charmed hadrons and the ratios given in the last section.

\section{B. Ratios of strange to nonstrange hadrons}

With Eqs. (33)-(36), we calculate yield ratios of strange to nonstrange charmed hadrons in $\mathrm{Au}$-Au collisions at $\sqrt{s_{\mathrm{NN}}}=$ $200 \mathrm{GeV}$. The results denoted by different curves are shown in Fig. 2. We see that the results are basically consistent with the data [15]. We also see that the enhancement of $D_{s}^{+} / D^{0}$ in $\mathrm{Au}-\mathrm{Au}$ collisions compared to those in $\mathrm{pp}$ reactions at $\mathrm{LHC}$ 

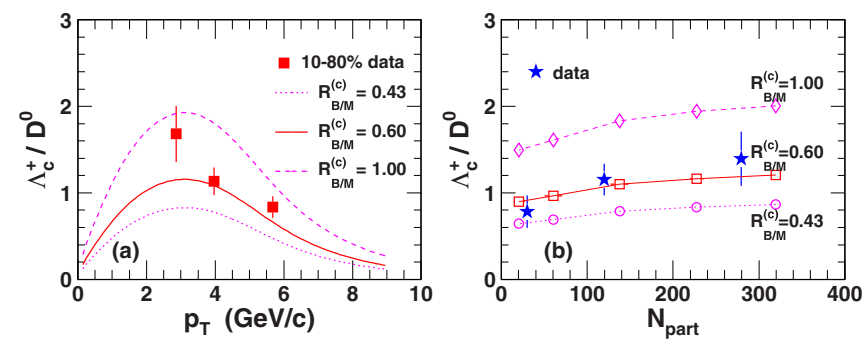

FIG. 3. (a) $\Lambda_{c}^{+} / D^{0}$ as a function of $p_{T}$ and (b) the $p_{T}$-integrated $\Lambda_{c}^{+} / D^{0}$ in the $p_{T}$ range from 3 to $6 \mathrm{GeV}$ as a function of $N_{\text {part }}$ in $\mathrm{Au}-\mathrm{Au}$ collisions at $\sqrt{s_{\mathrm{NN}}}=200 \mathrm{GeV}$. Solid squares and stars are the data from Ref. [14]. Different lines in panel (a) and open circles, squares, and rhombuses connected with the dotted, solid, and dashed lines to guide the eye in panel (b) are the calculated results with different values of $R_{B / M}^{(c)}$.

[47] comes mainly from the strangeness suppression factor $\lambda_{s}$ of the partonic matter in heavy ion collisions.

We also present the results for $\Xi_{c}^{+} / \Lambda_{c}^{+}, \Omega_{c}^{0} / \Xi_{c}^{0}$, and $\Omega_{c}^{0} / \Lambda_{c}^{+}$in Fig. 2. To see the effects of quark spectra in Eqs. (33)-(36) on these ratios, we plot also the constant factors in the corresponding panel in Fig. 2, respectively. We see that quark distributions slightly enhance these hadron yield ratios at the intermediate- $p_{T}$ region, but just the opposite at low- $p_{T}$ area.

\section{Baryon-to-meson ratios}

To calculate baryon-to-meson ratios in the charm sector, we need the parameter $R_{B / M}^{(c)}$ to determine $\mathcal{A}_{B} / \mathcal{A}_{M}$ in Eqs. (41)-(47). It was fixed as $R_{B / M}^{(c)}=0.43$ in $p p$ and $p$-Pb reactions at the energies available at LHC $[35,36]$. In heavy ion collisions its value may be larger due to the baryon-beneficial environment. To study the effect of $R_{B / M}^{(c)}$, we present results of calculations on $\Lambda_{c}^{+} / D^{0}$ in Fig. 3 with $R_{B / M}^{(c)}=0.43,0.60$, and 1.00 , respectively.

From Fig. 3, we see, just as expected, that $\Lambda_{c}^{+} / D^{0}$ shows a quite significant $p_{T}$ dependence, and the qualitative feature is consistent with the data available [14]. We also see that $R_{B / M}^{(c)}=0.60$ seems to be a suitable result for heavy ion collisions at top energies available at RHIC. In the following calculations of this article, we show only results obtained with this value.

To further study influences of quark distributions on baryon productions, we calculate the $p_{T}$ dependence of $\Lambda_{c}^{+} / D^{0}$ in different centralities in Au-Au collisions at $\sqrt{s_{\mathrm{NN}}}=200 \mathrm{GeV}$. The results are given in Fig. 4. We see that they all exhibit similar rise-peak-fall behaviors. From central to peripheral collisions, peak values decrease from about 1.3 to 1.0 , and the locations shift to lower $p_{T}$. This is due to the centrality dependence of $p_{T}$ distributions of quarks shown in Fig. 1, especially charm quarks. Other models, such as the Catania model [29] that includes coalescence and fragmentation, also give the rise-peak-fall behavior of $\Lambda_{c}^{+} / D^{0}$ as the function of $p_{T}$. However, the Catania model [29] predicts much flatter rising behavior at low- $p_{T}$ regions and almost no shift of peak location from energies available at RHIC to those available
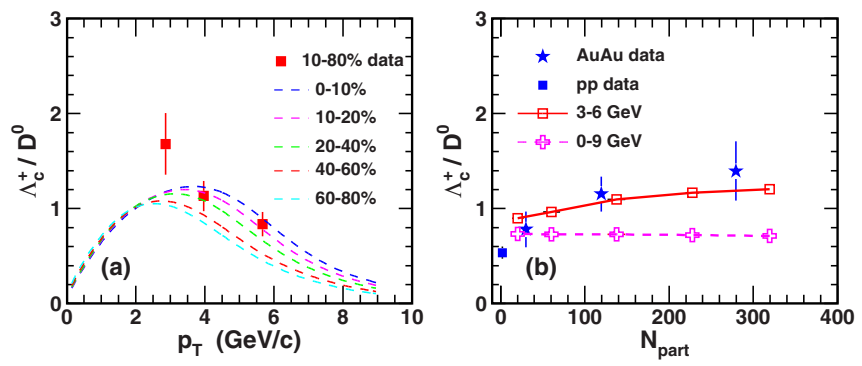

FIG. 4. (a) $\Lambda_{c}^{+} / D^{0}$ as a function of $p_{T}$ and (b) the $p_{T}$-integrated $\Lambda_{c}^{+} / D^{0}$ as a function of $N_{\text {part }}$ in Au-Au collisions at $\sqrt{s_{\mathrm{NN}}}=$ $200 \mathrm{GeV}$. The data are taken from Refs. $[14,18]$. In panel (b), the $\mathrm{Au}-\mathrm{Au}$ data are for $p_{T}$ in the range from 3 to $6 \mathrm{GeV}$ and the $p p$ data are for $p_{T}$ in the range from 3 to $4 \mathrm{GeV}$.

at LHC. Experimental measurements can distinguish these different models and provide important insights into charm quark hadronization in high-energy collisions.

Data of the $p_{T}$-integrated $\Lambda_{c}^{+} / D^{0}$ from central to peripheral $\mathrm{Au}-\mathrm{Au}$ collisions at $\sqrt{s_{\mathrm{NN}}}=200 \mathrm{GeV}$ show a decreasing trend [14] that is different from in light sectors [48] where baryon-to-meson ratios show little centrality dependence. We see that the calculated results in the same $p_{T}$ range (open squares in the figure) exhibit indeed such a trend consistent with the data [14]. However, if we take $p_{T}$ integrated from 0 to $9 \mathrm{GeV}$ (shown by the open crosses in the figure), this trend disappears and the result is essentially independent of the centrality and lower than those integrated from 3 to $6 \mathrm{GeV}$. Such properties come from the centrality dependence of $c$-quark distributions given by Fig. 1, where we see a strong dependence for larger $p_{T}$ but negligible dependence in the small- $p_{T}$ region. The results for $p_{T}$ integrated from 0 to $9 \mathrm{GeV}$ are dominated by the small $p_{T}$ contributions.

Encouraged by the agreements with data available, we make predictions for other similar baryon-to-meson ratios. The results are given in Figs. 5 and 6.

In Fig. 5, we see that all the ratios exhibit similar risepeak-fall behaviors as functions of $p_{T}$, and the peak locations change from central to peripheral collisions similar to $\Lambda_{c}^{+} / D^{0}$. In Fig. 6, we see similar trends for all these $p_{T}$-integrated ratios; i.e., they all show increasing tendencies for results integrated in the $p_{T}$ region from 3 to $6 \mathrm{GeV}$ but almost flat tendencies if integrated from 0 to $9 \mathrm{GeV}$.

At the end of this part, we would also like to emphasize that in our calculations, not merely $\Lambda_{c}^{+}$, but all charmed baryons are enhanced according to the overall ratio $R_{B / M}^{(c)}$ that was taken as 0.60 in the calculations. This is different from other coalescence models where diquarks were introduced to intensely enhance the production of $\Lambda_{c}^{+}$, but to less enhance or even not enhance other charmed baryons [25]. Future measurements of different charmed baryons should be very helpful in distinguishing different models and understanding the enhancement mechanism of charmed baryon production.

\section{The $p_{T}$ spectra of charmed hadrons}

Having the $p_{T}$ distributions of quarks, we not only calculate the ratios presented above but also the $p_{T}$ spectra of 

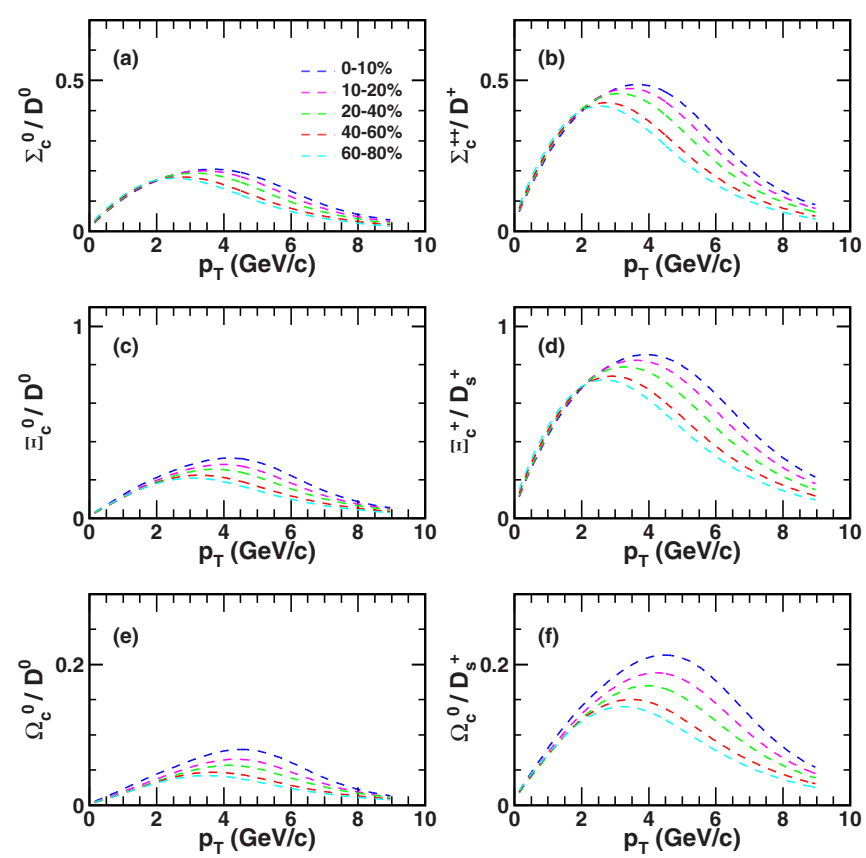

FIG. 5. Different baryon-to-meson ratios (a) $\Sigma_{c}^{0} / D^{0}$, (b) $\Sigma_{c}^{++} / D^{+}$, (c) $\Xi_{c}^{0} / D^{0}$, (d) $\Xi_{c}^{+} / D_{s}^{+}$, (e) $\Omega_{c}^{0} / D^{0}$, and (f) $\Omega_{c}^{0} / D_{s}^{+}$as functions of $p_{T}$ in different centralities in $\mathrm{Au}-\mathrm{Au}$ collisions at $\sqrt{s_{\mathrm{NN}}}=200 \mathrm{GeV}$.
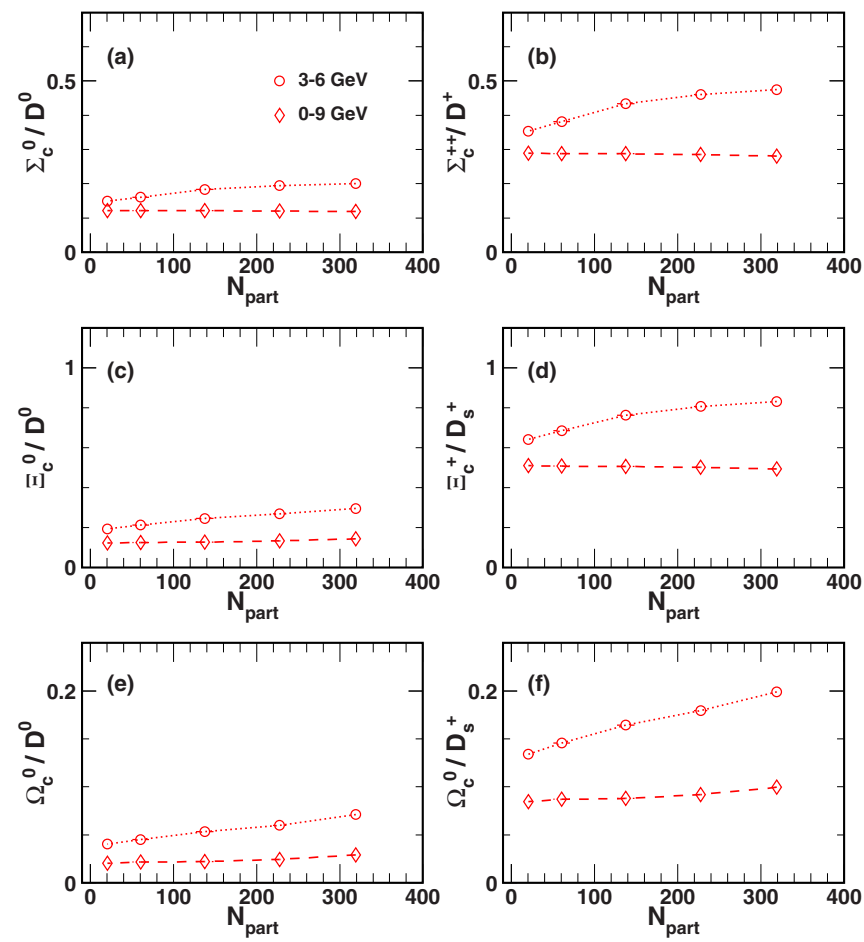

FIG. 6. Different $p_{T}$-integrated baryon-to-meson ratios (a) $\Sigma_{c}^{0} / D^{0}$, (b) $\Sigma_{c}^{++} / D^{+}$, (c) $\Xi_{c}^{0} / D^{0}$, (d) $\Xi_{c}^{+} / D_{s}^{+}$, (e) $\Omega_{c}^{0} / D^{0}$, and (f) $\Omega_{c}^{0} / D_{s}^{+}$as the function of $N_{\text {part }}$ in Au-Au collisions at $\sqrt{s_{\mathrm{NN}}}=200$ $\mathrm{GeV}$. Open circles are for $p_{T}$ integrated from 3 to $6 \mathrm{GeV}$ and open rhombuses are for $p_{T}$ integrated from 0 to $9 \mathrm{GeV}$.
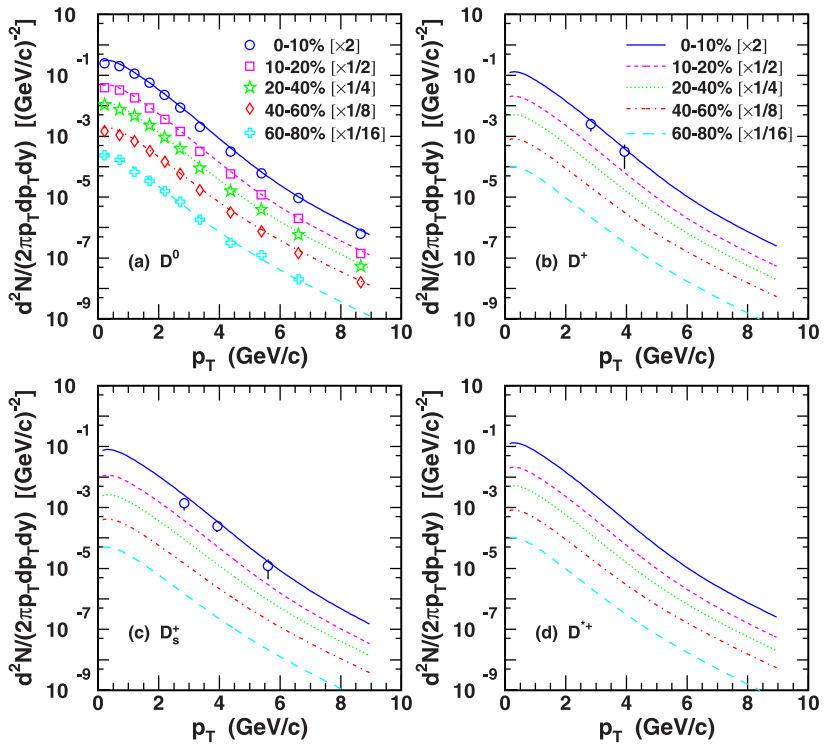

FIG. 7. $p_{T}$ spectra of open charm mesons (a) $D^{0}$, (b) $D^{+}$, (c) $D_{s}^{+}$, and (d) $D^{*+}$ in Au-Au collisions at $\sqrt{s_{\mathrm{NN}}}=200 \mathrm{GeV}$ in different centralities. The open symbols are data taken from Refs. [12,15].

charmed hadrons obtained under EVC. We present the results in the following. To obtain not only the shape but also the magnitudes, we need the rapidity density of charm quarks $d N_{c} / d y($ at $y=0)$ as an input. For this purpose, we estimate it by extrapolating $p p$ reaction data on the differential cross section $d \sigma_{c}^{p p} / d y$ and take $d N_{c} / d y=\left\langle T_{A A}\right\rangle d \sigma_{c}^{p p} / d y$, where $\left\langle T_{A A}\right\rangle$ is the average nuclear overlap function and can be calculated by the Glauber model $[49,50]$. We use $d \sigma_{c}^{p p} / d y=$ $130 \pm 30 \pm 26 \mu \mathrm{b}$ recently measured at midrapidity in $p p$ at $\sqrt{s}=200 \mathrm{GeV}$ [14], and we obtain $d N_{c} / d y=2.945 \pm$ $0.680 \pm 0.589$ in the most-central $0-10 \%$ collisions in $\mathrm{Au}-$ $\mathrm{Au}$ collisions at $\sqrt{s_{\mathrm{NN}}}=200 \mathrm{GeV}$. Considering the data of $D^{0}$ [12], we take $d N_{c} / d y=2.45$ for the centrality $0-10 \%$. For the other centralities, 10-20\%, 20-40\%, 40-60\%, and $60-80 \%$, we have $d N_{c} / d y=1.54,0.76,0.24$, and 0.055 , respectively.

In Fig. 7, we present $p_{T}$ spectra of different $D$ mesons in $\mathrm{Au}-\mathrm{Au}$ collisions at $\sqrt{s_{\mathrm{NN}}}=200 \mathrm{GeV}$ together with data

TABLE II. Yield densities $d N / d y$ of charmed hadrons in the midrapidity in different centralities in Au-Au collisions at $\sqrt{s_{\mathrm{NN}}}=$ $200 \mathrm{GeV}$.

\begin{tabular}{llllll}
\hline \hline Hadron & $0-10 \%$ & $10-20 \%$ & $20-40 \%$ & $40-60 \%$ & $60-80 \%$ \\
\hline$D^{0}$ & 0.893 & 0.570 & 0.284 & 0.0898 & 0.0207 \\
$D^{+}$ & 0.377 & 0.241 & 0.120 & 0.0379 & 0.00874 \\
$D^{*+}$ & 0.381 & 0.243 & 0.121 & 0.0383 & 0.00883 \\
$D_{s}^{+}$ & 0.261 & 0.151 & 0.0713 & 0.0223 & 0.00495 \\
$\Lambda_{c}^{+}$ & 0.636 & 0.412 & 0.207 & 0.0655 & 0.0152 \\
$\Sigma_{c}^{0}$ & 0.106 & 0.0687 & 0.0344 & 0.0109 & 0.00253 \\
$\Sigma_{c}^{++}$ & 0.106 & 0.0687 & 0.0344 & 0.0109 & 0.00253 \\
$\Xi_{c}^{0}$ & 0.129 & 0.0758 & 0.0360 & 0.0113 & 0.00252 \\
$\Xi_{c}^{+}$ & 0.129 & 0.0758 & 0.0360 & 0.0113 & 0.00252 \\
$\Omega_{c}^{0}$ & 0.0260 & 0.0139 & 0.00626 & 0.00194 & 0.000419 \\
\hline \hline
\end{tabular}



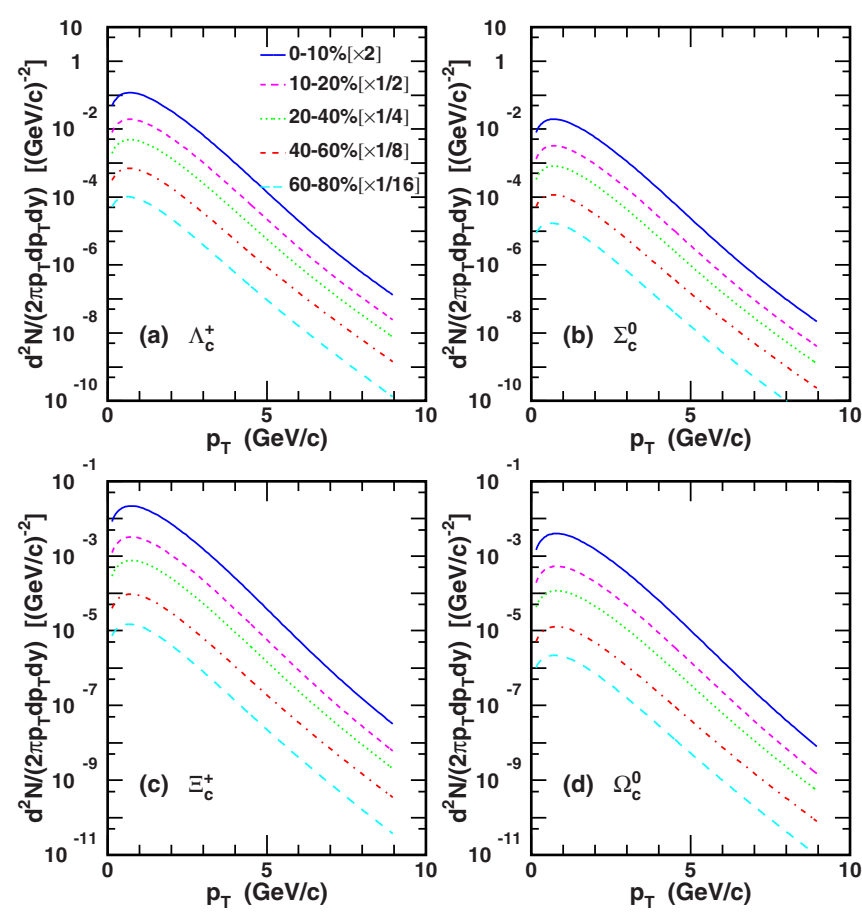

FIG. 8. Calculated results for $p_{T}$ spectra of singly charmed baryons (a) $\Lambda_{c}^{+}$, (b) $\Sigma_{c}^{0}$, (c) $\Xi_{c}^{+}$, and (d) $\Omega_{c}^{0}$ in different centralities in $\mathrm{Au}-\mathrm{Au}$ collisions at $\sqrt{s_{\mathrm{NN}}}=200 \mathrm{GeV}$.

available $[12,15]$. We see that the results agree reasonably with the data. In Fig. 8, we show results for different charmed baryons. In Table II, we also present $p_{T}$-integrated yield densities $d N / d y$ of different charmed hadrons at the midrapidity in different centralities. These results can all be used to test the hadronization mechanisms, in particular EVC, in future experiments.

\section{SUMMARY}

Though not much data are available yet, charm hadron production seems to provide an important test of different hadronization mechanisms in heavy ion collisions. In this article, we have derived the $p_{T}$ dependence of open charm mesons and singly charmed baryons in the quark combination model under the EVC in ultrarelativistic heavy ion collisions. We present in particular analytic expressions of two groups of hadron yield ratios, the strange to nonstrange charmed hadron ratios and baryon-to-meson ratios in terms of normalized $p_{T}$ spectra of quarks. We present normalized $p_{T}$ spectra of quarks and numerical results for these hadron yield ratios using these quark $p_{T}$ spectra. We found that the magnitude of the strange to nonstrange charmed hadron ratios are mainly determined by the strangeness suppression factor and have weak $p_{T}$ dependencies. In contrast, there is an obvious $p_{T}$ dependence for baryon-to-meson ratios determined by the quark $p_{T}$ spectra. The different baryon-to-meson ratios have similar $p_{T}$ and centrality dependencies sensitive to $p_{T}$ distribution of $c$ quark. We have compared the results obtained with the data available and present predictions for future experiments. Further studies along this line can provide more sensitive tests of charm quark hadronization mechanisms and insight on properties of the QGP in heavy ion collisions.

\section{ACKNOWLEDGMENTS}

We thank Zhang-Bu Xu for helpful discussions. This work was supported in part by the National Natural Science Foundation of China under Grants No. 11505104, No. 11575100, No. 11675092 and No. 11975011 and by the Natural Science Foundation of Shandong Province, China, under Grant No. ZR2019YQ06.
[1] A. Andronic et al., Eur. Phys. J. C 76, 107 (2016).

[2] F. Prino and R. Rapp, J. Phys. G: Nucl. Part. Phys. 43, 093002 (2016).

[3] R. Rapp and H. van Hees, in Quark-Gluon Plasma 4, edited by R. C. Hwa and X.-N. Wang (World Scientific, Singapore, 2010).

[4] N. Armesto, A. Dainese, C. A. Salgado, and U. A. Wiedemann, Phys. Rev. D 71, 054027 (2005).

[5] Y. L. Dokshitzer and D. E. Kharzeev, Phys. Lett. B 519, 199 (2001).

[6] P. Braun-Munzinger, V. Koch, T. Schäfer, and J. Stachel, Phys. Rep. 621, 76 (2016).

[7] S. Batsouli, S. Kelly, M. Gyulassy, and J. L. Nagle, Phys. Lett. B 557, 26 (2003).

[8] N. Xu and Z. Xu, Nucl. Phys. A 715, 587 (2003).

[9] Z. W. Lin and D. Molnar, Phys. Rev. C 68, 044901 (2003).

[10] G. D. Moore and D. Teaney, Phys. Rev. C 71, 064904 (2005).

[11] H. van Hees, V. Greco, and R. Rapp, Phys. Rev. C 73, 034913 (2006).

[12] J. Adam et al. (STAR Collaboration), Phys. Rev. C 99, 034908 (2019).

[13] S. Acharya et al. (ALICE Collaboration), J. High Energy Phys. 10 (2018) 174.
[14] S. Radhakrishnan (STAR Collaboration), Nucl. Phys. A 982, 659 (2019).

[15] L. Zhou (STAR Collaboration), Nucl. Phys. A 967, 620 (2017).

[16] S. Acharya et al. (ALICE Collaboration), J. High Energy Phys. 12 (2019) 092.

[17] S. Acharya et al. (ALICE Collaboration), Phys. Lett. B 793, 212 (2019).

[18] S. Acharya et al. (ALICE Collaboration), J. High Energy Phys. 04 (2018) 108.

[19] M. Lisovyi, A. Verbytskyi, and O. Zenaiev, Eur. Phys. J. C 76, 397 (2016).

[20] T. Sjostrand, S. Mrenna, and P. Skands, J. High Energy Phys. 05 (2006) 026.

[21] R. Maciula and A. Szczurek, Phys. Rev. D 98, 014016 (2018).

[22] A. Andronic, P. Braun-Munzinger, K. Redlich, and J. Stachel, Phys. Lett. B 659, 149 (2008).

[23] I. Kuznetsova and J. Rafelski, Eur. Phys. J. C 51, 113 (2007).

[24] V. Greco, C. M. Ko, and R. Rapp, Phys. Lett. B 595, 202 (2004).

[25] S. H. Lee, K. Ohnishi, S. Yasui, I.-K. Yoo, and C. M. Ko, Phys. Rev. Lett. 100, 222301 (2008). 
[26] Y. P. Liu, C. Greiner, and A. Kostyuk, Phys. Rev. C 87, 014910 (2013).

[27] M. He, R. J. Fries, and R. Rapp, Phys. Rev. C 86, 014903 (2012).

[28] H. J. Xu, X. Dong, L. J. Ruan, Q. Wang, Z. B. Xu, and Y. F. Zhang, Phys. Rev. C 89, 024905 (2014).

[29] S. Plumari, V. Minissale, S. K. Das, G. Coci, and V. Greco, Eur. Phys. J. C 78, 348 (2018).

[30] J. Zhao, S. Shi, N. Xu, and P. Zhuang, arXiv:1805.10858.

[31] M. He and R. Rapp, Phys. Rev. Lett. 124, 042301 (2020).

[32] S. Cho, K.-J. Sun, C. M. Ko, S. H. Lee, and Y. Oh, Phys. Rev. C 101, 024909 (2020).

[33] R. Q. Wang, J. Song, and F. L. Shao, Phys. Rev. C 91, 014909 (2015).

[34] J. Song, X. R. Gou, F. L. Shao, and Z. T. Liang, Phys. Lett. B 774, 516 (2017); X. R. Gou, F. L. Shao, R. Q. Wang, H. H. Li, and J. Song, Phys. Rev. D 96, 094010 (2017).

[35] H.-h. Li, F.-1. Shao, J. Song, and R.-q. Wang, Phys. Rev. C 97, 064915 (2018).

[36] J. Song, H.-h. Li, and F.-1. Shao, Eur. Phys. J. C 78, 344 (2018).

[37] R. Q. Wang, F. L. Shao, J. Song, Q. B. Xie, and Z. T. Liang, Phys. Rev. C 86, 054906 (2012).

[38] S. Weinberg, The Quantum Theory of Fields (Cambridge University, Cambridge, England, 1995), p. 169.

[39] See, e.g., Q. B. Xie and Z. T. Liang, A hadronization model and its application to e+ $\mathrm{e}-$ annihilation, in Multiparticle
Production, Proceedings of the Shandong Workshop, Jinan, Shandong, China, June 28-July 6, 1987, edited by Rudolph C. Hwa and Xie Qu-bing (World Scientific, Jinan, Shandong, China, 1987), pp. 469-496; Q. B. Xie and X. M. Liu, Phys. Rev. D 38, 2169 (1988); F. L. Shao, Q. B. Xie, and Q. Wang, Phys. Rev. C 71, 044903 (2005).

[40] X. Dong, Y.-J. Lee, and R. Rapp, Annu. Rev. Nucl. Part. Sci. 69, 417 (2019).

[41] M. Tanabashi et al. (Particle Data Group), Phys. Rev. D 98, 030001 (2018).

[42] B. Abelev et al. (ALICE Collaboration), J. High Energy Phys. 07 (2012) 191.

[43] S. S. Adler et al. (PHENIX Collaboration), Phys. Rev. C 69, 034909 (2004).

[44] J. Adams et al. (STAR Collaboration), Phys. Rev. Lett. 98, 062301 (2007).

[45] B. I. Abelev et al. (STAR Collaboration), Phys. Rev. Lett. 99, 112301 (2007).

[46] F. Scardina, S. K. Das, V. Minissale, S. Plumari, and V. Greco, Phys. Rev. C 96, 044905 (2017).

[47] S. Acharya et al. (ALICE Collaboration), Eur. Phys. J. C 77, 550 (2017).

[48] F.-1. Shao, G.-j. Wang, R.-q. Wang, H.-h. Li, and J. Song, Phys. Rev. C 95, 064911 (2017), and experimental references therein.

[49] R. J. Glauber and G. Matthiae, Nucl. Phys. B 21, 135 (1970).

[50] C. Loizides, J. Kamin, and D. d'Enterria, Phys. Rev. C 97, 054910 (2018). 